\title{
Notes on Remainders of Paratopological Groups*
}

\author{
Hanfeng Wang Wei He
}

\begin{abstract}
In this paper, it is proved that a non-locally compact paratopological group $G$ has a remainder which is a $p$-space if and only if $G$ is either a Lindelöf $p$-space or a $\sigma$-compact space. We show that if $G$ is a non-locally compact paratopological group with a compactification $b G$ such that the remainder $b G \backslash G$ is locally metrizable, then both $G$ and $b G$ are separable and metrizable. It is proved that if $G$ is a cosmic paratopological group with a paracompact remainder, then $G$ is separable and metrizable.
\end{abstract}

\section{Introduction}

By a remainder of a Tychonoff topological space $G$, we mean the subspace $b G \backslash G$ of some compactification $b G$ of $G$. Remainders of a topological group or a paratopological group have many interesting properties and have been studied extensively in literature (see [1]-[6] and [8]-[11]).

One of the most interesting questions in the study of remainders is to determine to what extent a property of a topological space $X$ is related to another property of some or all remainders of $X$. A classical result about remainders is the following theorem due to M. Henriksen and J. Isbell [18]:

Theorem 1.1. A Tychonoff space $X$ is of countable type if and only if the remainder in any (or some) Hausdorff compactification of $X$ is Lindelöf.

\footnotetext{
${ }^{*}$ Project supported by NSFC (11171156)

Received by the editors in February 2013 - In revised form in November 2013.

Communicated by E. Colebunders.

2010 Mathematics Subject Classification : 54D40, 54E35, 22A05.

Key words and phrases : remainder; paratopological group; compactification; metrizable; $p$-space; $\pi$-character.
} 
In [11] Arhangel'skii studied properties of topological groups with an Ohio complete remainder. He proved that a non-locally compact topological group $G$ has a remainder which is a $p$-space if and only if $G$ is either a Lindelöf $p$-space or a $\sigma$-compact space.

In this paper we generalize the above result and prove the following:

A non-locally compact paratopological group $G$ has a remainder which is a $p$-space if and only if $G$ is either a Lindelöf $p$-space or a $\sigma$-compact space.

We investigate local metrizability of remainders of a paratopological group. We prove that if $G$ is a non-locally compact paratopological group with a compactification $b G$ such that the remainder $b G \backslash G$ is locally metrizable, then both $G$ and $b G$ are separable and metrizable.

We show that a cosmic paratopological group with a paracompact remainder must be separable and metrizable.

We also investigate remainders of semitopological groups. It is proved that if a separable semitopological group $G$ has a remainder $Y$ with countable $\pi$-character, then either $Y$ is countably compact, or $G$ has a countable $\pi$-base.

Throughout this paper, a topological space always means a Tychonoff space. $c(X)$ is the cellularity or Souslin number of the space $X$. For unexplained terms and symbols we refer the reader to [7] or [14].

\section{Preliminaries}

Recall that a topological group $G$ is a group $G$ with a topology such that multiplication on $G$ considered as a map of $G \times G$ to $G$ is jointly continuous and the inversion in $G$ is continuous. A paratopological group $G$ is a group $G$ with a topology such that multiplication on $G$ is jointly continuous. A semitopological group $G$ is a group $G$ with a topology such that multiplication on $G$ is separately continuous.

Recall that a space $X$ is of (pointwise) countable type if every (point) compact subset $P$ of $X$ is contained in a compact subset $F \subset X$ that has a countable base of open neighbourhoods in X. Obviously, every space of countable type is of pointwise countable type. However, the converse is not true, even in the category of homogeneous spaces [12]. So the following result by A.V. Arhangel'skii is interesting.

Theorem 2.1. [2] Let $G$ be a paratopological group. If there exists a non-empty compact subset of $G$ of countable character in $G$, then $G$ is a space of countable type.

From Theorem 2.1 we know that a paratopological group of pointwise countable type is a space of countable type.

Let $\mathcal{O}$ be a family of open subsets of a space $X$ and $F$ be a subset of $X$. $\mathcal{O}$ is said to be an outer base of $F$ in $X$ if for each $x \in F$ and each neighbourhood $U$ of $x$ in $X$ there exists an element $V$ of $\mathcal{O}$ such that $x \in V \subset U$.

Recall that a space $X$ is called Ohio complete [11], if in every compactification $b X$ of $X$ there exists a $G_{\delta}$-subset $Z$ such that $X \subset Z$ and every $y \in Z \backslash X$ is separated from $X$ by a $G_{\delta}$-subset of $Z$. By [11] all $p$-spaces and all Lindelöf spaces are Ohio complete. 


\section{Remainders of paratopological groups}

To extend Arhangel'skii's result mentioned in the introduction to paratopological groups, we first prove a lemma.

Lemma 3.1. Let $G$ be a homogeneous space with a compactification $b G$ such that the remainder $b G \backslash G$ is Ohio complete. Then either $b G \backslash G$ is Čech-complete or $G$ is of pointwise countable type.

Proof We consider two cases.

Case 1: $G$ is locally compact. Then $G$ is of countable type and $b G \backslash G$ is compact.

Case 2: $G$ is non-locally compact. Then $G$ is nowhere locally compact, since $G$ is a homogeneous space. It follows that the remainder $Y=b G \backslash G$ is dense in $b G$. Hence, $b G$ is also a compactification of $b G \backslash G$. Since $b G \backslash G$ is Ohio complete, we can fix a $G_{\delta}$-subset $Z$ of $b G$ such that $Y \subset Z$ and every $y \in Z \backslash Y$ can be separated from $Y$ by a $G_{\delta}$-subset of $Z$.

If $Z \backslash Y$ is empty, then $Y=Z$ is a $G_{\delta}$-subset $Z$ of $b G$ which implies that $b G \backslash G$ is Čech-complete.

If $Z \backslash Y$ is not empty, one can take a point $p \in Z \backslash Y$ and a $G_{\delta}$-subset $P$ of $Z$ such that $p \in P \subset Z \backslash Y$. Then $p \in P \subset G$, and $P$ is a $G_{\delta}$-subset of $b G$ since $Z$ is a $G_{\delta}$-subset of $b G$. Since $b G$ is compact, it follows that there exists a non-empty compact subset $F \subset P$ such that $F$ has a countable base of open neighbourhoods in $G$. Since $G$ is a homogeneous space, $G$ has a cover by compact subsets with countable bases of open neighbourhoods in $G$. Then it follows that $G$ is of pointwise countable type.

Lemma 3.2. [11] If $X$ is a Lindelöf $p$-space, then any remainder of $X$ is a Lindelöf $p$ space.

Theorem 3.1. Suppose that $G$ is a non-locally compact paratopological group and that $b G$ is a compactification of $G$. Then the remainder $b G \backslash G$ is a $p$-space if and only if at least one of the following conditions holds:

(1) $G$ is a Lindelöf $p$-space;

(2) $G$ is $\sigma$-compact.

Proof Sufficiency: If $G$ is a Lindelöf $p$-space, then by Lemma 3.2, $b G \backslash G$ is a Lindelöf $p$-space. If $G$ is $\sigma$-compact, then $b G \backslash G$ is Cech-complete, which implies that $b G \backslash G$ is a $p$-space.

Necessity. Since every $p$-space is Ohio complete [11], it follows from Lemma 3.1 that either $b G \backslash G$ is Cech-complete or $G$ is of pointwise countable type. If $b G \backslash G$ is Čech-complete, then $G$ is $\sigma$-compact. If $G$ is of pointwise countable type, then $G$ is of countable type by Theorem 2.1. It follows that $b G \backslash G$ is Lindelöf, by Theorem 1.1. Since $b G \backslash G$ is a Lindelöf $p$-space, Lemma 3.2 implies that $G$ is a Lindelöf $p$-space.

Corollary 3.1. Suppose that $G$ is a non-locally compact paratopological group with a compactification $b G$ such that the remainder $b G \backslash G$ is a paracompact $p$-space. Then $G$ is a Lindelöf p-space. 
Proof By Theorem 3.1, G is a Lindelöf $p$-space or a $\sigma$-compact space. Suppose $G$ is $\sigma$-compact. The cellularity of a $\sigma$-compact paratopological group is countable [7, Corollary 5.7.12]. It follows that $c(G) \leq \omega$. Since $G$ is dense in $b G, c(b G) \leq$ $\omega$. It follows that $c(b G \backslash G) \leq \omega$, since $b G \backslash G$ is dense in $b G$. Thus, $b G \backslash G$ is Lindelöf, since $b G \backslash G$ is paracompact and $c(b G \backslash G) \leq \omega$. Since $b G \backslash G$ is a Lindelöf $p$-space and $G$ is a remainder of $b G \backslash G, G$ is a Lindelöf $p$-space by Lemma 3.2.

By Corollary 3.1 we have the following result.

Corollary 3.2. Suppose that $G$ is a non-locally compact paratopological group with a compactification $b G$ such that the remainder $b G \backslash G$ is a paracompact $p$-space. Then both $G$ and $b G \backslash G$ are Lindelöf $p$-spaces.

Corollary 3.3. Let $G$ be a paratopological group with a $G_{\delta^{-}}$diagonal and let $b G$ be a compactification of $G$. If the remainder $b G \backslash G$ is a paracompact p-space, then $G$ is metrizable.

Proof If $G$ is locally compact, then $G$ is a topological group since a locally compact paratopological group is a topological group [7, Proposition 2.3.11]. Since every locally compact space is a $p$-space, it follows that $G$ is a paracompact $p$-space [7, Theorem 4.3.35]. It follows that $G$ is metrizable since $G$ has a $G_{\delta}$-diagonal [15].

If $G$ is non-locally compact, then $G$ is a Lindelöf $p$-space by Corollary 3.1. Since $G$ has a $G_{\delta}$-diagonal, it follows that $G$ is separable and metrizable [15].

Corollary 3.4. Let $G$ be a paratopological group with a $G_{\delta^{-}}$diagonal and let $b G$ be a compactification of $G$. If the remainder $b G \backslash G$ is a $p$-space, then $G$ is a cosmic space. In particular, $G$ is submetrizable.

Proof By Theorem 3.1, G is a Lindelöf $p$-space or $\sigma$-compact. If $G$ is a Lindelöf $p$-space with a $G_{\delta}$-diagonal, then $G$ is separable and metrizable, which implies that $G$ has a countable network. If $G$ is $\sigma$-compact, $G$ has a countable network, since every compact subspace with a $G_{\delta}$-diagonal is separable and metrizable [15].

Since every semitopological group with countable $\pi$-character has a $G_{\delta}$-diagonal [7, Corollary 5.7.5], by Corollary 3.4, we have the following result.

Corollary 3.5. Let $G$ be a paratopological group with countable $\pi$-character and let $b G$ be a compactification of $G$. If the remainder $b G \backslash G$ is a p-space, then $G$ is a cosmic space.

Theorem 3.2. Let $G$ be a cosmic paratopological group with a compactification $b G$ such that the remainder $b G \backslash G$ is paracompact, then $G$ is separable and metrizable.

Proof If $G$ is locally compact, then $G$ is a $p$-space. Since $G$ is a cosmic space, $G$ is a Lindelöf space with a $G_{\delta}$-diagonal. Since every Lindelöf $p$-space with a $G_{\delta}$-diagonal is separable and metrizable, so is $G$.

If $G$ is not locally compact, then $b G \backslash G$ is dense in $b G$ and $G$ is a remainder of $b G \backslash G$. Then we have $c(G) \leq \omega$ since $G$ has a countable network. Since $G$ is dense in $b G, c(b G) \leq \omega$. It follows that $c(b G \backslash G) \leq \omega$, since $b G \backslash G$ is dense in $b G$. Thus, $b G \backslash G$ is Lindelöf, since it is paracompact and $c(b G \backslash G) \leq \omega$. 
Then $G$ is of countable type, by Theorem 1.1. Therefore, there exists a compact subset $K \subset G$ such that $K$ has a countable base of open neighbourhoods in $G$. Since $K$ is compact and has a countable network, it follows that $K$ is separable and metrizable [14]. By [17], a compact subspace $F$ of a space $X$, such that $F$ is separable and metrizable and has a countable base of open neighbourhoods in $X$, has a countable outer base in $X$. Therefore, $K$ has a countable outer base in $G$. In particular, $G$ has a countable local base at every point of $K$. Since $G$ is homogeneous, it follows that $G$ is first countable. Since $G$ is a paratopological group, it has a countable base by a result of Ravsky (see [19, Proposition 2.13]). Therefore, $G$ is separable and metrizable.

In Theorem 3.2, the condition that $G$ has a countable network cannot be replaced by the weaker one that $G$ has a countable $\pi$-base. Indeed, the Sorgenfrey line $G$, as a paratopological group with a countable $\pi$-base, has a compactification $b G$ which is homeomorphic to the two arrows space, and the remainder $b G \backslash G$ is Lindelöf. However, $G$ is non-metrizable.

In [10], Arhangel'skii proved that if a non-locally compact topological group has a compactification $b G$ such that the remainder $b G \backslash G$ has a $G_{\delta}$-diagonal, then both $G$ and $b G$ are separable and metrizable. However, the conclusion is false in the category of paratopological groups [10]. By Corollary 3.2, we have the following result.

Theorem 3.3. Let $G$ be a non-locally compact paratopological group with a compactification $b G$ such that the remainder $b G \backslash G$ is metrizable. Then both $G$ and $b G$ are separable and metrizable.

Proof Since $b G \backslash G$ is metrizable, it is a paracompact $p$-space. By Corollary 3.2, both $G$ and $b G \backslash G$ are Lindelöf $p$-spaces. Since $b G \backslash G$ is metrizable and Lindelöf, it follows that $b G \backslash G$ is separable and metrizable.

Fix a countable base $\mathcal{B}$ of $b G \backslash G$. For each $B \in \mathcal{B}$, take an open subset $V_{B}$ of $b G$ such that $V_{B} \cap(b G \backslash G)=B$. Put $O_{B}=V_{B} \cap G$, for each $B \in \mathcal{B}$. Since both $b G \backslash G$ and $G$ are dense in $b G$, it follows that $\left\{O_{B}: B \in \mathcal{B}\right\}$ is a countable $\pi$-base of $G$. Since a paratopological group with countable $\pi$-character has a $G_{\delta}$-diagonal [7, Corollary 5.7.5], $G$ has a $G_{\delta}$-diagonal. Therefore, $G$ is separable and metrizable, since $G$ is a Lindelöf $p$-space with a $G_{\delta}$-diagonal [15].

Since both $G$ and $b G \backslash G$ are separable and metrizable, it follows that $b G$ has a countable network. Therefore, $b G$ is separable and metrizable.

In [4] C. Liu studied local properties of remainders of a topological group and proved that if a non-locally compact topological group has a compactification $b G$ such that $b G \backslash G$ has a local $G_{\delta}$-diagonal, then both $G$ and $b G$ are separable and metrizable. For non-locally compact paratopological groups with locally metrizable remainders, we can show the following result which complements Theorem 3.3.

Theorem 3.4. Suppose $G$ is a non-locally compact paratopological group with a compactification $b G$ such that the remainder $b G \backslash G$ is locally metrizable. Then both $G$ and $b G$ are separable and metrizable. 
Proof Fix a point $y \in b G \backslash G$ and two open neighbourhoods $V_{y}$ and $W_{y}$ of $y$ in $b G \backslash G$ such that $W_{y}$ is metrizable and the closure of $V_{y}$ in $b G \backslash G$ is contained in $W_{y}$. We denote by $U_{y}$ the closure of $V_{y}$ in $b G \backslash G$. Obviously, $U_{y}$ is metrizable.

Claim 1: $U_{y}$ is not countably compact. Suppose to the contrary that $U_{y}$ is countably compact. Then $U_{y}$ is is compact, since $U_{y}$ is metrizable. Hence, $U_{y}$ is closed in $b G$. On the other hand, there exists an open neighbourhood $U$ of $y$ in $b G$ such that $U \cap(b G \backslash G)=V_{y}$. Since $G$ is non-locally compact and homogeneous, $G$ is nowhere locally compact, which implies that $b G \backslash G$ is dense in $b G$. Therefore, $V_{y}$ is dense in $U$. Thus, the closure of $U$ in $b G$ coincides with the closure of $V_{y}$ in $b G$. It follows that the closure of $U$ in $b G$ coincides with $U_{y}$. This contradicts the fact that $U \cap G \neq \varnothing$.

Claim 2: $G$ has a $G_{\delta}$-diagonal. Since $U_{y}$ is not countably compact, there exists an infinite closed discrete countable subset $F$ of $b G \backslash G$ contained in $U_{y}$. Since $b G$ is compact, there exists a point $c$ in $G$ such that $c \in \bar{F}^{b G}$. Since $W_{y}$ is an open subset of $b G \backslash G$ such that that $W_{y}$ is metrizable and $U_{y} \subset W_{y}$, it follows that $b G \backslash G$ has countable character at each point of $F$. Then $b G$ has countable character at each point of $F$, since $b G \backslash G$ is dense in $b G$. For each $y \in F$, take a countable local base $\mathcal{O}_{y}$ of $y$ in $b G$, and put $\mathcal{B}_{y}=\left\{V \cap G: V \in \mathcal{O}_{y}\right\}$. Then $\bigcup_{y \in F} \mathcal{B}_{y}$ is a countable $\pi$-base of $c$ in $G$. Since $G$ is homogeneous, $G$ has countable $\pi$-character. It follows that $G$ has a $G_{\delta}$-diagonal [7, Corollary 5.7.5].

Let $K$ be the closure of $U_{y}$ in $b G$. Obviously, $K \backslash U_{y}$ is a non-empty subset of $G$, and the interior of $K \backslash U_{y}$ in $G$ is also not empty. Since $U_{y}$ is metrizable, it is Ohio complete [11]. Therefore, there exists a $G_{\delta}$-subset $H$ of $K$ such that $U_{y} \subset H$ and every $x \in H \backslash U_{y}$ is separated from $U_{y}$ by a $G_{\delta}$-subset of $H$.

Now we show that both $G$ and $b G$ are separable and metrizable. For this purpose, we consider two cases.

Case 1: $H \backslash U_{y}=\varnothing$, i.e. $H=U_{y}$. Then $K \backslash U_{y}$ is $\sigma$-compact. Since $K \backslash U_{y}$ is contained in $G, K \backslash U_{y}$ has a $G_{\delta}$-diagonal. Thus, $K \backslash U_{y}$ has a countable network, which implies that $c\left(K \backslash U_{y}\right) \leq \omega$. Therefore, $c(K) \leq \omega$, since $K \backslash U_{y}$ is dense in $K$. It follows from the density of $U_{y}$ in $K$ that $c\left(U_{y}\right) \leq \omega$. Therefore, $U_{y}$ is separable and metrizable. Since both $U_{y}$ and $K \backslash U_{y}$ have countable networks, it follows that $K$ has a countable network. Thus, $K$ is separable and metrizable. Then $K \backslash U_{y}$ is separable and metrizable. Since $G$ is homogeneous, it follows that $G$ is locally separable and locally metrizable.

We claim that $G$ is of countable type. Take an arbitrary compact subset $C$ of $G$. For every $x \in C$, fix an open neighbourhood $O_{x}$ of $x$ in $G$ such that $O_{x}$ is separable and metrizable. Then there exists a finite subset $A$ of $G$ such that $C \subset \bigcup\left\{O_{x}: x \in A\right\}$. It follows that $C$ has a countable outer base in $G$. Then it is easy to see that $C$ has a countable character in $G$. Thus, $G$ is of countable type.

By Theorem 1.1, $b G \backslash G$ is Lindelöf. Hence $b G \backslash G$ is locally separable since $b G \backslash G$ is Lindelöf and locally metrizable. Since $b G \backslash G$ is locally separable and locally metrizable, it follows that $b G \backslash G$ is separable and metrizable. Therefore, $b G \backslash G$ is a Lindelöf $p$-space. Then $G$ is a Lindelöf $p$-space by Corollary 3.2. Since $G$ has a $G_{\delta}$-diagonal, it follows that $G$ is separable and metrizable. Since both $b G \backslash G$ and $G$ are separable and metrizable, one can conclude that $b G$ is separable and metrizable. 
Case 2: $H \backslash U_{y} \neq \varnothing$. Let $O$ be the interior of $K \backslash U_{y}$ in $G$. Then $O$ is dense in $K$. Since $G$ is dense in $b G$, it follows that $O \subseteq \operatorname{Int}_{b G} K$. We have the following two subcases.

Subcase (a): $H \cap O=\varnothing$. Then $O \subset K \backslash H$, which implies that $K \backslash H$ is dense in $K$. Since $K \backslash H$ is $\sigma$-compact and has a $G_{\delta}$-diagonal, it follows that $K \backslash H$ has a countable network. Thus $c(K \backslash H) \leq \omega$. Since both $K \backslash H$ and $U_{y}$ are dense in $K$, one can conclude that $c\left(U_{y}\right) \leq \omega$. Therefore, $U_{y}$ is separable and metrizable. Then $K \backslash U_{y}$ is a Lindelöf $p$-space. It follows from the fact that $K \backslash U_{y}$ has a $G_{\delta}$-diagonal that $K \backslash U_{y}$ is separable and metrizable, which implies that $G$ is locally separable and locally metrizable. As in Case 1, we come to the conclusion that both $G$ and $b G$ are separable and metrizable.

Subcase (b): $H \cap O \neq \varnothing$. Fix a point $x \in H \cap O$, then there is a $G_{\delta}$-subset $P$ of $H$ such that $x \in P \subset H \backslash U_{y}$. Since $H$ is a $G_{\delta}$-subset of $K$, it follows that $P$ is a $G_{\delta}$-subset of $K$. Let $\left\{P_{n}: n \in \omega\right\}$ be a sequence of open subsets of $K$ such that $P=\cap\left\{P_{n}: n \in \omega\right\}$. Take a sequence $\left\{W_{n}: n \in \omega\right\}$ of open neighbourhoods of $x$ in $b G$ such that $W_{0} \subset K$ and $\overline{W_{n+1}} \subset W_{n} \cap P_{n}$. It is easy to see that $\left\{W_{n}: n \in \omega\right\}$ is a local base of the compact set $\cap\left\{W_{n}: n \in \omega\right\}$ in $b G$. Obviously, $\cap\left\{W_{n}: n \in \omega\right\}$ is contained in $G$ and has countable character in $G$. Since $G$ is a paratopological group, it follows that $G$ is of countable type, by Theorem 2.1. Therefore, $b G \backslash G$ is Lindelöf. Since $b G \backslash G$ is locally metrizable, it is locally separable. It follows that $b G \backslash G$ is separable and metrizable. As in Case 1, this implies that both $G$ and $b G$ are separable and metrizable.

Next we consider semitopological groups with remainders of countable $\pi$ character.

Theorem 3.5. Suppose $G$ is a non-locally compact separable semitopological group with a compactification $b G$ such that the remainder $b G \backslash G$ has countable $\pi$-character. Then either $b G \backslash G$ is countably compact or $G$ has a countable $\pi$-base.

Proof Assume that $b G \backslash G$ is not countably compact. Then there exists a countable infinite closed discrete subset $F$ of $b G \backslash G$. Since $b G$ is compact, there exists a point $p$ of $G$ such that $p \in \bar{F}^{b G}$. Since $G$ is non-locally compact, $b G \backslash G$ is dense in $b G$. Then it follows from the fact that $b G \backslash G$ has countable $\pi$-character that each point of $b G \backslash G$ has a countable $\pi$-base in $b G$. For each $y \in F$, take a countable $\pi$-base $\mathcal{O}_{y}$ of $y$ in $b G$, and put $\mathcal{B}_{y}=\left\{V \cap G: V \in \mathcal{O}_{y}\right\}$. Then $\bigcup_{y \in F} \mathcal{B}_{y}$ is a countable $\pi$-base of $p$ in $G$.

Since $G$ is homogeneous, $G$ has a countable $\pi$-base $\mathcal{B}_{e}$ at the identity $e$. Take a countable subset $L$ of $G$ such that $L$ is dense in $G$. Put $\mathcal{B}=\left\{x U: x \in L, U \in \mathcal{B}_{e}\right\}$. We claim that $\mathcal{B}$ is a countable $\pi$-base of $G$.

Indeed, for each point $a$ of $G$ and an open neighbourhood $W$ of $a$, there exists a point $x$ of $L$ such that $x \in W$. Since $G$ is a semitopological group, there exists a neighbourhood $V$ of $e$ such that $x V \subset W$. Since $\mathcal{B}_{e}$ is a $\pi$-base of $G$ at $e$, we can find an element $U \in \mathcal{B}_{e}$ such that $U \subset V$. Then $x U \in \mathcal{B}$ and $x U \subset W$. Therefore, $\mathcal{B}$ is a countable $\pi$-base of $G$.

Theorem 3.6. Suppose $G$ is a non-locally compact cosmic semitopological group with a compactification $b G$ such that the remainder $b G \backslash G$ has countable $\pi$-character. Then either $b G \backslash G$ is Čech-complete or $G$ has a countable $\pi$-base. 
Proof Let $\mathcal{N}$ be a countable closed network of $G$. Denote by $\gamma$ the family of all compact elements of $\mathcal{N}$. We consider two cases.

Case 1: $\bigcup \gamma=G$. It follows that $G$ is $\sigma$-compact, which implies that $b G \backslash G$ is Cech-complete.

Case 2: $G \backslash \cup \gamma \neq \varnothing$. Fix a point $a \in G \backslash \cup \gamma$ and put $\beta=\{P \in \mathcal{N}: a \in$ $P\}$. Then $\beta$ is a countable network of $G$ at $a$, and none element of $\beta$ is compact. Therefore, $\bar{P}^{b G} \cap(b G \backslash G) \neq \varnothing$, for each $P \in \beta$. Fix a point $y_{P} \in \bar{P}^{b G} \cap(b G \backslash G)$ for each $P \in \beta$, and put $A=\left\{y_{P}: P \in \beta\right\}$. Then $A$ is countable and $a \in \bar{A}^{b G}$. Since $b G \backslash G$ is dense in $b G$ and has countable $\pi$-character, it follows that $b G$ has countable $\pi$-character at every point of $b G \backslash G$. For each $y_{P} \in A$, take a countable $\pi$-base $\mathcal{O}_{P}$ of $y_{P}$ in $b G$, and put $\mathcal{B}_{P}=\left\{V \cap G: V \in \mathcal{O}_{P}\right\}$. Obviously, $\bigcup_{y_{P} \in A} \mathcal{B}_{P}$ is a countable $\pi$-base of $a$ in $G$. Since $G$ is homogeneous, $G$ has a countable $\pi$-base $\mathcal{B}_{e}$ at the identity $e$. Since $G$ has a countable network, it is separable. Let $S$ be a countable subset of $G$ which is dense in $G$, and put $\mathcal{V}=\left\{s A: s \in S, A \in \mathcal{B}_{e}\right\}$. Then it follows from the proof of Theorem 3.5 that $\mathcal{V}$ is a $\pi$-base of $G$.

Recall that a Tychonoff space $X$ is said to be weakly pseudocompact if there exists a Hausdorff compactification $b X$ such that $X$ is $G_{\delta}$-dense in $b X$, that is, every non-empty $G_{\delta}$-set in $b X$ intersects $X$.

Theorem 3.7. Suppose $G$ is a weakly pseudocompact semitopological group with a compactification $b G$ such that the remainder $b G \backslash G$ has countable $\pi$-character. Then either $b G \backslash G$ is countably compact or $G$ is a topological group metrizable by a complete metric.

Proof Suppose that $b G \backslash G$ is not countably compact. Then $b G \backslash G$ is not compact and, hence, $G$ is not locally compact. As in the proof of Theorem 3.5, we see that $G$ has countable $\pi$-character. Then $G$ has a $G_{\delta}$-diagonal. However, every weakly pseudocompact Tychonoff space $X$ with a $G_{\delta}$-diagonal is Čech-complete [7, Proposition 5.7.19]. Further, every Čech-complete semitopological group is a topological group [13]. Then $G$ is a topological group with countable $\pi$-character, which implies that $G$ is metrizable [16]. Since $G$ is Cech-complete, it follows that $G$ is a completely metrizable topological group.

Since every pseudocompact space is weakly pseudocompact and pseudocompact metrizable space is compact, the following result follows from Theorem 3.7 .

Corollary 3.6. Suppose that $G$ is a pseudocompact and non-compact semitopological group and $b G$ is a compactification of $G$. If the remainder $b G \backslash G$ has countable $\pi$-character, then $b G \backslash G$ is countably compact.

\section{Acknowledgment}

The authors would like to thank the referee for his/her valuable comments and suggestions to improve the paper. 


\section{References}

[1] C. Liu, Metrizability of paratopological (semitopological) groups, Topology Appl. 159 (2012), 1415-1420.

[2] A.V. Arhangel'skii, M.M. Choban, On remainders of rectifiable spaces, Topology Appl. 157 (2010), 789-799.

[3] A.V. Arhangel'skii, $G_{\delta}$-points in remainders of topological groups and some addition theorems in compacta, Topology Appl. 156 (2009), 2013-2018.

[4] C. Liu, Remainders in compactifications of topological groups, Topology Appl. 156 (2009), 849-854.

[5] D. Basile, A. Bella, About remainders in compactifications of homogeneous spaces, Comment.Math.Univ.Carolin. 50,4 (2009), 607-613.

[6] A.V. Arhangel'skii, The Baire properties in remainders of topological groups and other results, Comment.Math.Univ.Carolin. 50,2 (2009), 273-279.

[7] A.V. Arhangel'skii, M. Tkachenko, Topological Groups and Related Structures, Atlantis Press, Amsterdam-Paris, 2008.

[8] A.V. Arhangel'skii, Two types of remainders of topological groups, Comment. Math.Univ.Carolin. 49,1 (2008), 119-126.

[9] A.V. Arhangel'skii, First countability, tightness, and other cardinal invariants in remainders of topological groups, Topology Appl. 154 (2007), 29502961.

[10] A.V. Arhangel'skii, More on remainders close to metrizable spaces, Topology Appl. 154 (2007), 1084-1088.

[11] A.V. Arhangel'skii, Remainders in compactifications and generalized metrizability properties, Topology Appl. 150 (2005), 79-90.

[12] D. Basile, J.Van Mill, A homogeneous space of point-countable but not of countable type, Comment.Math.Univ.Carolin. 48,3 (2007), 459-463.

[13] A. Bouziad, Every Čech-analytic Baire semitopological group is a topological group, Proc. Amer. Math. Soc. 24, 3 (1998), 953-959.

[14] R. Engelking, General Topology, Revised and completed edition, Heldermann Verlag Berlin, 1989.

[15] G. Gruenhage, Handbook of set-theoretic topology (K. Kunen and J.E. Vaughan, eds.), North-Holland, Amsterdam, 1984, 423-501.

[16] A.V. Arhangel'skii, Relations among the invariants of topological groups and their subspaces, Russian Math. Surveys, 35 (1980), 1-23.

[17] Y. Kodama, K. Nagami, General Topology, Iwanami Shoten, Tokyo, 1974. 
[18] M. Henriksen, J.R. Isbell, Some properties of compactifications, Duke Math. J. 25 (1958), 83-106.

[19] A.V. Arhangel'skii, E.V. Reznichenko, Paratopological and semitopological groups versus topological groups, Topology. Appl. 151 (2005), 107-119.

Institute of Mathematics,

Nanjing Normal University,

Nanjing 210046,

China

and

Department of Mathematics,

Shandong Agricultural University,

Taian 271018, China

E-mail address:weihe@njnu.edu.cn 